\title{
Chaotic behavior of triatomic clusters
}

\author{
Ersin Yurtsever* \\ Chemistry Department, Koç University, Istinye 80860, İstanbul, Turkey \\ Nuran Elmac1 \\ Chemistry Department, Middle East Technical University, Ankara, Turkey
}

(Received 26 April 1996)

\begin{abstract}
The dynamics of triatomic clusters is investigated employing two-body Lennard-Jones and three-body Axilrod-Teller potential functions. Lyapunov exponents are calculated for the total energy range of $-2.70 \epsilon$ $<E<-0.72 \epsilon$. The effects of the initial geometry of the cluster, its angular momentum, and the magnitude of three-body interactions are analyzed. It has been found that the dominating factor for the extent of chaotic behavior is the energy assigned to vibrational modes. The introduction of the rotational motion regularizes the dynamics in spite of a higher degree of nonlinearity. The three-body terms in the potential function affect the extent of the chaos in different manners depending on the initial geometry of the cluster. Finally, the time evolution of heterogeneous clusters generated by varying the size, mass, and the interaction strength of a single atom is observed. Their Lyapunov exponent spectra show that the additional nonlinearity reduces the chaotic behavior of the system in most of the cases. [S1050-2947(96)03012-0]

PACS number(s): 36.40.-c, 05.45.+b
\end{abstract}

\section{INTRODUCTION}

The dynamics of small systems poses interesting questions concerning the mechanisms of isomerization and/or fragmentation and time scales of these processes [1-14]. The form of the essential dynamics, the internal energy distribution to various modes (whether local or nonlocal), and the factors governing the fragmentation are crucial questions both for molecules and clusters. The basic difference between a cluster and a molecule can be explained in terms of the strengths of interactions between atoms. The potential energy hypersurface of a molecule usually displays welldefined minima and the molecules are trapped in these wells due to high-energy barriers separating them. Normal-modetype approximations for the truncation of potential energy functions are valid, and the dynamics can simply be treated as weakly coupled small-amplitude vibrations around the minima. When the interactions between atoms are weaker, the number of accessible optimum points along the hypersurfaces for the given energy increases drastically and the cluster moves between these points much more freely. This flexibility introduces a great degree of richness to the dynamics. From the mathematical point of view, the normal modes lose their meaning and the system can no longer be represented by a set of uncoupled oscillators. The classical equations of motion governing the dynamics are now highly nonlinear and one expects that their solutions should exhibit some degree of chaos.

The large majority of investigations of chaotic behavior of Hamiltonian systems are restricted to problems with only a few degrees of freedom, mostly because of the numerical complications in the computations of measures of chaos. The standard definition of chaos can be formulated in terms of the initial state dependence, that is, small perturbations to the

*Author to whom correspondence should be addressed. initial conditions can result in vastly different developments even in the near future. It is no longer possible to make predictions for the outcome of events. This sensitivity to initial conditions can also be expressed in terms of the rate of loss of information about the initial state of the system. The most commonly used criteria for observing this loss of information are Lyapunov exponents or Kolmogorov entropy, which is the sum of all positive Lyapunov exponents. The magnitude of positive Lyapunov exponents, especially the maximum one, is generally accepted as a unique and continuous measure driving the system from regular (insensitive to small perturbations) to chaotic dynamics. Usually an increase in the energy (Hamiltonian) is accompanied by an increase in the maximum Lyapunov exponent with certain critical values separating regular, transitional, and fully chaotic regimes. However, the boundaries between these regimes are not very sharp due to the complex structure of the phase space. Especially for systems with many degrees of freedom, it is possible that the classical phase space for the given energy is not of uniform nature, that is, not all trajectories with the same energy behave similarly. In that case, it may be necessary to find out the relative measures of each regime. This can be achieved by analyzing a bundle of trajectories that span the classical phase space as much as possible, and then classifying them according to their qualitative behavior. Such partitioning of the phase space is generally a property of its geometry and optimum points, hence a thorough knowledge of the potential energy hypersurface is required.

In the case of chaotic dynamics of clusters, there are relatively fewer reports and most of them by Berry and coworkers [15-23]. In these studies atomic clusters of various sizes that interact mostly via Lennard-Jones-type potentials are reported. The first large-scale calculations of such manydimensional problems were on the correlations of melting and phase transitions to fractal dimensions and Kolmogorov entropy [17]. Here it was noted that the topology of the po- 
tential energy hypersurface played an extremely important role in identifying chaos, and later the various forms of the dynamics were associated with saddle points of the surface. Another important contribution was the study of Lyapunov exponent distributions in order to understand the ergodicity of small clusters $[20,21]$. It was shown that the distribution of exponents contained a great deal of information since individual exponents cannot be used as direct measures of the ergodicity. Finally it was noted that the early evolution of Lyapunov exponents displayed significant information about the dynamics [19]. The standard practice in the computation of Lyapunov exponents is to discard the results obtained during the equilibration period and to study the limiting values. Even though this procedure is mathematically correct in identifying classical chaos, part of the physically relevant information is lost. All of these results point to the important role that the topology of the phase space plays in the recognition of chaos in cluster dynamics.

Our original interest was in the stability of carbon clusters and their fragmentation processes. The mechanisms for these processes involve a great deal of isomerizations, hence a thorough knowledge of the optimum points as well as the transitions between different structures is required. We have previously looked at very simple force fields that are combinations of Lennard-Jones and Axilrod-Teller-type potential functions and have shown that relative stability of various isomers of $\mathrm{C}_{3}-\mathrm{C}_{6}$ can be fairly predicted once the strength of the three-body interactions is optimized [24]. Therefore we decided to first follow the dynamics for $\mathrm{C}_{3}$ employing twobody interactions and to investigate the effects of the energy, initial geometry, and angular momentum. Later by adding three-body terms the changes in the characteristics of the dynamics were studied. Finally we varied the mass, size, and interaction parameters of a single atom in triatomic LennardJones clusters so that the chaotic behavior of heterogeneous clusters could be measured.

\section{COMPUTATIONAL METHOD}

\section{A. Lyapunov exponents}

The nonlinear dynamics of the cluster is followed by solving Hamilton's equations of motion with a constant-timestep-Runge-Kutta integration of the fourth order. The choice of the Runge-Kutta over the faster predictor-corrector methods is due to the difficulty in keeping the energy constant at the very early stages of the integration. Since for such methods, the higher derivatives of the velocity are taken as zero at $t=0$, the energy may fluctuate slightly. However, this error is not expected to affect the results, at least qualitatively. The tangent space method is used for computing Lyapunov exponents. An infinitesimal $6 \mathrm{~N}$-dimensional hyperellipsoid is placed at the initial conditions in phase space and the time evolution of its axes is followed. Tangent space is defined in order to avoid the exponential divergence of these axes; that is, the equations of motion are written for the differences between the phase-space vector defining the cluster and each vector corresponding to the axes of the hyperellipsoid. Since these differences are small, they can be expressed as Taylor series and keeping only the first terms, we end up with linearized equations for the time evolution of each of these axes. We denote the vector function for the nonlinear dynamics as $F(x(t))$ with the definition

$$
\begin{aligned}
F(x(t))= & \left(\partial q_{1} / \partial t, \partial q_{2} / \partial t, \ldots, \partial q_{3 N} / \partial t,\right. \\
& \left.\times \partial p_{1} / \partial t, \partial p_{2} / \partial t, \ldots, \partial p_{3 N} / \partial t\right),
\end{aligned}
$$

which is explicitly given as

$$
\begin{aligned}
F(x(t))= & \left(p_{1}, p_{2}, \ldots, p_{3 N},-\partial H / \partial q_{1},-\partial H / \partial q_{2}, \ldots,\right. \\
& \left.-\partial H / \partial q_{3 N}\right)
\end{aligned}
$$

with $q$ and $p$ representing the coordinates and momenta of each atom. The linearized equations for the principal axes are then written as

$$
d \delta x^{k}(t) / d t=\partial F(x(t)) /\left.\partial x\right|_{x=x(t)} \delta x^{k}(t)
$$

in which $\delta x^{k}$ denotes the vector containing $6 \mathrm{~N}$ elements of the $k$ th principal axis. Since these equations are linear, the initial vectors can be taken as finite vectors, and in fact they are normalized to 1 . By following these vectors, the phase space in the vicinity of the vector $x(t)$ is probed. Like $x(t)$, $\delta x^{k}(t)$ also diverges exponentially in case of chaotic dynamics. Therefore they have to be orthogonalized at regular intervals. Each time we orthonormalize the vectors, we record the length of each axis which, in turn, gives the corresponding Lyapunov exponent as

$$
\lambda_{k}=\lim t^{-1} \ln d_{k}(t) / d_{k}(0) .
$$

Here $d_{k}$ is the length of the $k$ th axis before orthonormalization. The frequency of the Gram-Schmidt orthonormalization does not really affect the results.

Equation (3) must be solved simultaneously with Hamilton's equations for $F(x(t))$. Runge-Kutta integration requires the computation of the Hamiltonian's derivatives four times at every time step. At each of these steps, the Jacobian has to be modified according to the intermediate values of the vector $F$.

\section{B. Computations}

Hamilton's equations of motion are solved for $10^{6}$ steps for each trajectory. At small intervals they are stored so that results can be compared to those from thermally equilibrated clusters. The integrations are carried out in atomic units and the constant time step is chosen as 50 a.u., which is in the order of $10^{-3}$ ps. This time step is found as the largest interval, which keeps the energy constant to about six to seven digits. However, for the various step sizes considered, Lyapunov exponents display, qualitatively, similar behavior. Of course, due to the highly chaotic nature of the dynamics, the actual values differ to an extent. At every 10 steps, the length of each vector is computed and then orthonormalized. The Lyapunov exponents converge sufficiently well in the integration time of $1200 \mathrm{ps}$. The integration and computation of the full set of Lyapunov exponents of a single trajectory takes about 40 min on a SGI workstation Indigo R4000 or 10 min on R8000.

The initial geometry of the structure is chosen either as an equilateral triangle (minimum energy conformation for Lennard-Jones system) or as a linear one (global minimum 
once the three-body term gains sufficient strength). The momentum components of each particle are assigned randomly with the condition that the center of mass does not move. To identify the effects of the angular momentum, we compared clusters of the same energy with both zero and nonzero angular momentum. The nonrotating clusters are prepared in a brute force Monte Carlo fashion. Random momenta are assigned to each atom with zero total linear momentum. Then random components are changed slightly, the momentum of the center of mass is subtracted and then resulting angular momentum is calculated to see whether it is decreased or not. After several cycles of these processes, clusters with total angular momentum of $10^{-6}$ a.u. can be obtained. Afterwards momenta are scaled to the desired energy.

In this work the energy of the cluster is always expressed in units of $N \epsilon$, where $\epsilon$ is the interaction parameter of the Lennard-Jones potential and $N$ is the number of atoms. The potential-energy expression up to three-body interactions is

$$
E=\frac{1}{2} \sum V_{\mathrm{LJ}}(i, j)+V_{\mathrm{AT}}(i, j, k),
$$

where the summation runs over all atoms. For two-body interactions only, the potential-energy function takes the form of the Lennard-Jones potential:

$$
V_{\mathrm{LJ}}(i, j)=4 \epsilon\left[\left(\sigma / r_{i j}\right)^{12}-\left(\sigma / r_{i j}\right)^{6}\right] .
$$

In terms of these units the minimum potential energy is -1.0 for the equilateral triangle and -0.67 for the linear one. Above $E=-0.33$ the cluster may dissociate. Upon dissociation, the system turns into a one-dimensional problem of a diatomic cluster plus a free atom; therefore the dynamics quickly becomes a regular one. All these clusters that dissociate are excluded from any averaging process. The energy range from -0.60 ( -0.90 for triangular case) to -0.24 is studied with varying numbers of initial conditions.

To represent three-body interactions, we employ the Axilrod-Teller functional form which is written as

$$
V_{\mathrm{AT}}=Z\left(1+3 \cos \Theta_{i} \cos \Theta_{i} \cos \Theta_{k}\right) r_{i j}^{-3} r_{i k}^{-3} r_{j k}^{-3},
$$

with $r$ and $\Theta$ corresponding to the sides and angles of the triangle formed by atoms $i, j$, and $k$. The interaction strength is controlled by a single parameter $Z$ whose unit is $4 \epsilon \sigma^{9}$.

For homonuclear clusters, the dynamics can be studied independently of two parameters, $\epsilon$ and $\sigma$ of the LennardJones function (or similarly the interaction strength $Z$ of the Axilrod-Teller). However, since we ultimately would like to study carbon clusters, we choose two sets of parameters that are used in the simulations of carbon containing compounds with mass being 12.0 proton mass unit. In the first set (denoted by I) they are given as $\epsilon=0.70656 \times 10^{-21} \mathrm{~J}$ and $\sigma=3.35 \AA$. These parameters are used to simulate liquid $\mathrm{CS}_{2}$ [25]. The second set (II) consists of $\epsilon=9.9496 \times 10^{-19} \mathrm{~J}$ and $\sigma=1.1047144 \AA$, which is obtained both from ab initio calculations and from spectroscopic observations [26]. Set I is used in all calculations with two-body forces and set II is used whenever three-body terms are included. The threebody interaction strength $Z$ is varied from 0.0 to 1.0 in units of $4 \epsilon \sigma^{9}$.

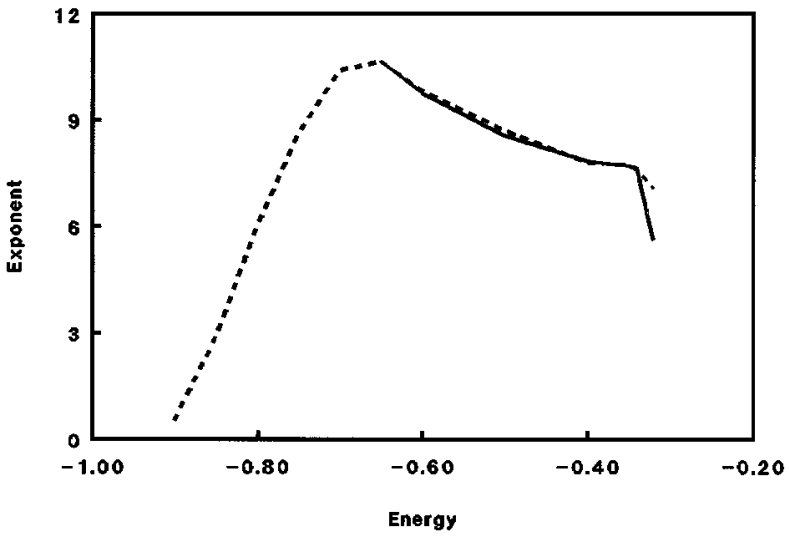

FIG. 1. Maximum Lyapunov exponent as a function of the total energy for nonrotating clusters. _- linear; ----, triangular.

\section{RESULTS AND DISCUSSION}

\section{A. Angular momentum}

Since a thorough search of an 18-dimensional phase space is very difficult, we try a rough partitioning based on two initial geometrical structures, 16 energy values, and two sets of 50 random initial momenta corresponding to rotating and nonrotating clusters. Half of the trajectories start from an equilateral triangle, which is the global minimum for the Lennard-Jones cluster with corresponding interatomic distances of $2^{1 / 6} \sigma$. The other half start from the minimum energy conformation of the linear cluster in which the distances between adjacent atoms are slightly shorter than those of the triangular form.

The same initial seed for the random number generator is used for different sets to sample the momentum space so that relatively similar parts of the phase space are spanned, although due to the differences in the geometric structure and consequently the potential energy as well as the total energy, each trajectory still originates from different initial conditions. The angular momentum is conserved during integration; therefore trajectories with zero or nonzero angular momentum visit different parts of the phase space. In this manner we are able to study the effects of the angular momentum on chaotic behavior.

In Fig. 1 we present the variation of the maximum Lyapunov exponents as a function of the energy for linear and triangular nonrotating clusters. Exponents reported here are averages over 50 trajectories. The low-energy regime below $E=-0.67$ is not accessible in the linear cluster so a comparison cannot be made. As it was shown in Berry's work, the maximum Lyapunov exponent is very close to zero at the bottom of the well, displaying regular dynamics due to the high separability of the nuclear motion (normal modes are valid). As the energy increases, the chaos starts to settle in up to the saddle point for the triatomic Lennard-Jones system. Then, again, we observe a gradual decrease in the Lyapunov exponent. At the high-energy regime, both curves for linear and triangular clusters agree well showing that an isomerization takes place at early stages and the initial geometry does not really affect the dynamics if no rotational motion exists.

In contrast, the rotating clusters display significantly different behavior for linear and triangular structures (Fig. 2). 


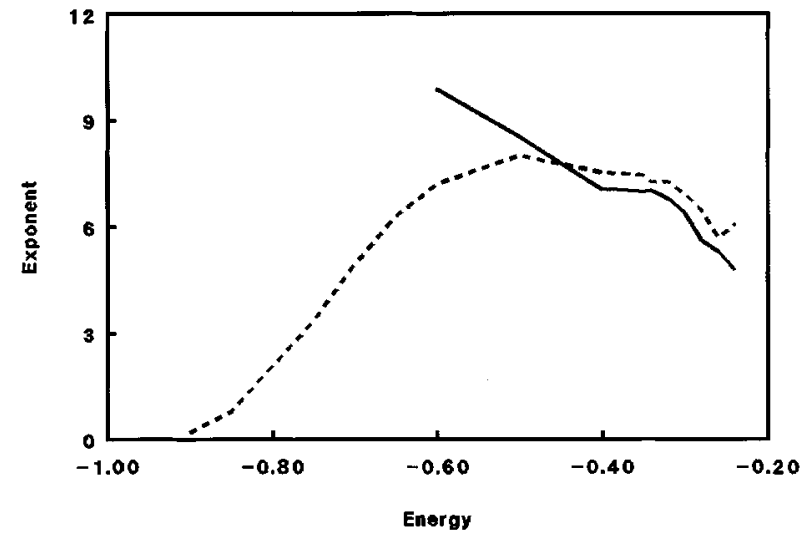

FIG. 2. Maximum Lyapunov exponent as a function of the total energy for rotating clusters. _—, linear; ----, triangular.

At low-energy values, initially linear clusters have higher Lyapunov exponents. There is a crossing point around $E=$ -0.45 above which the linear clusters are less chaotic. Before we assign any significance to the roles of the initial geometry in generating chaos, it is necessary to analyze how the angular momentum is affecting the dynamics. For the nonrotating clusters, kinetic energy is only present in the vibrational degrees of freedom and the rotational kinetic energy is zero. Therefore it is clear that any degree of chaos could only result from the vibrational motion. In the case of the rotating clusters, the situation is different. Since the momentum components for each atom are selected randomly (only scaled to preset energy values), the components and the total angular momentum of each cluster in the sample are different. Consequently the partitioning of the initial kinetic energy assigned to each cluster in terms of rotational and vibrational terms varies throughout the bundle of trajectories.

Now there are two possible sources of the nonlinearity and hence chaotic behavior in the dynamics. First of all, individual magnitudes of the vibrational and rotational energy may be important. For rigid bodies, rotational kinetic energy is constant, so it does not play any role in the classification of the motion. In case of a nonrigid body, even though this partitioning does not remain constant but rather is a function of time, it can nevertheless be a driving factor

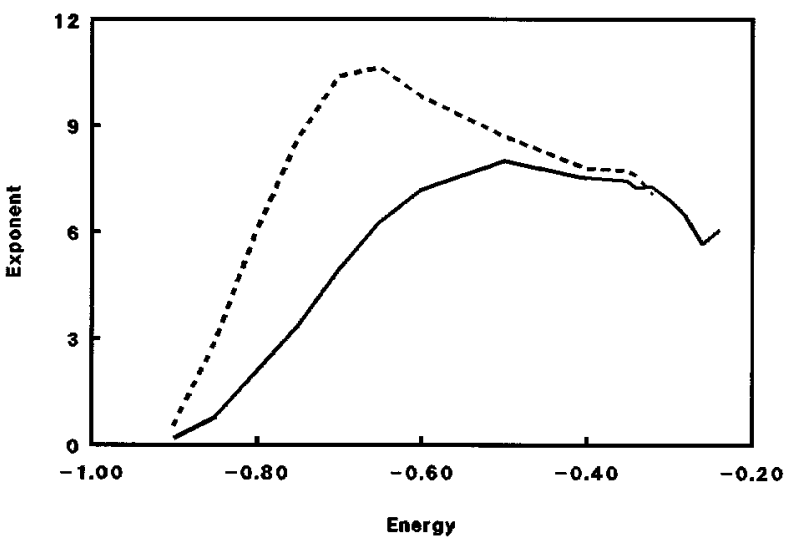

FIG. 3. Maximum Lyapunov exponent as a function of the total energy for clusters initiated as equilateral triangles. _ _ rotating; ----, nonrotating.
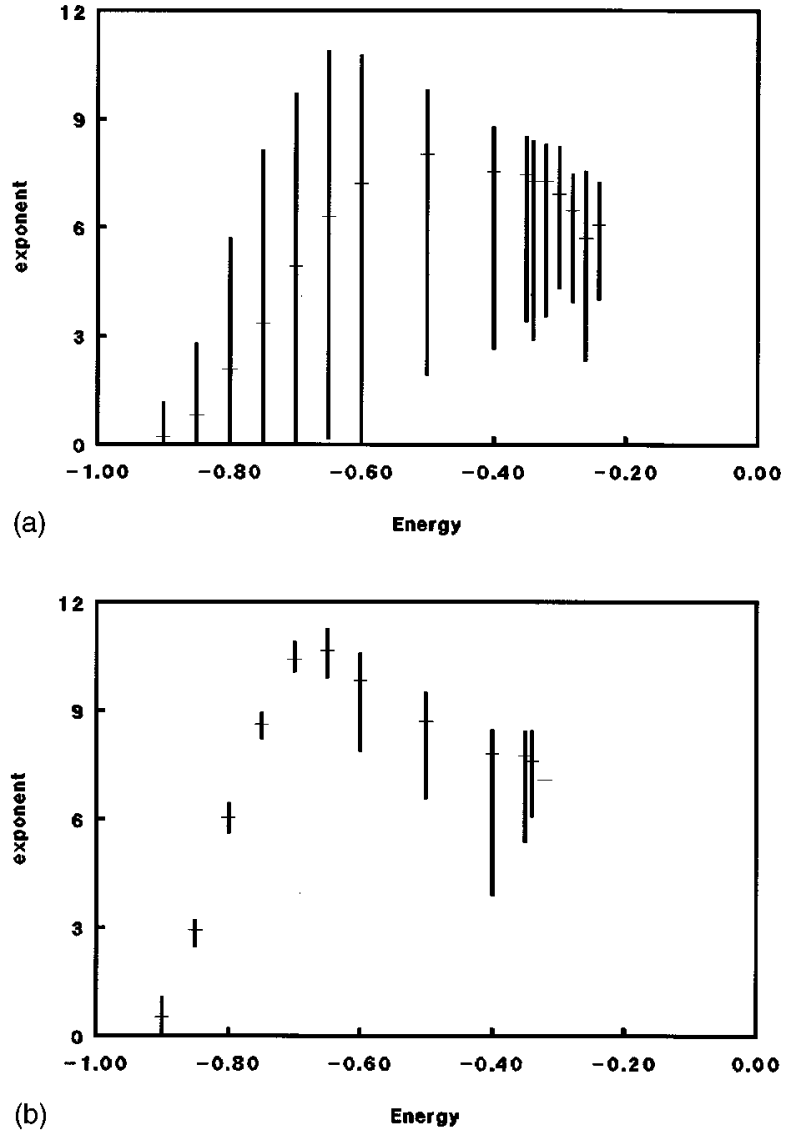

FIG. 4. Variation of Lyapunov exponents within an energy shell. (a) Rotating, (b) Nonrotating clusters.

for the dynamics. Another source of nonlinearity may come from the coupling between the vibrational and rotational motion. Since any such coupling would bring additional complexity to the problem, it should increase the chaotic character. In Fig. 3 the average exponents for the rotating and nonrotating clusters initiating from the triangular structure are displayed to analyze these two somewhat contrasting factors. At high-energy values above $E=-0.4$ the rotational motion does not play any role in the dynamics, and both curves agree well. One should be careful in this region since the excess vibrational energy usually drives the cluster to fragmentation and those trajectories must be excluded from the averaging process. However, there are significant differences for the low-energy regime even around the almost harmonic part of the potential.

The maximum Lyapunov exponents corresponding to nonrotating clusters are always larger than those for nonrotating ones with ratios as large as two. In contrast to the nonrotating cluster displaying a maximum around the saddle point, there are no optimum points for the rotational case except at very-high energy values. In this region there seem to be fluctuations most likely due to the smaller number of nondissociating trajectories. It is clear that the coupling between the rotational and the vibrational motion does not really contribute to chaotic behavior and the dominating factor is the available vibrational energy.

In Fig. 4, the variations of Lyapunov exponents obtained from 50 trajectories are given for the triangular geometry. Initially, all the nonrotating clusters at the same energy have 

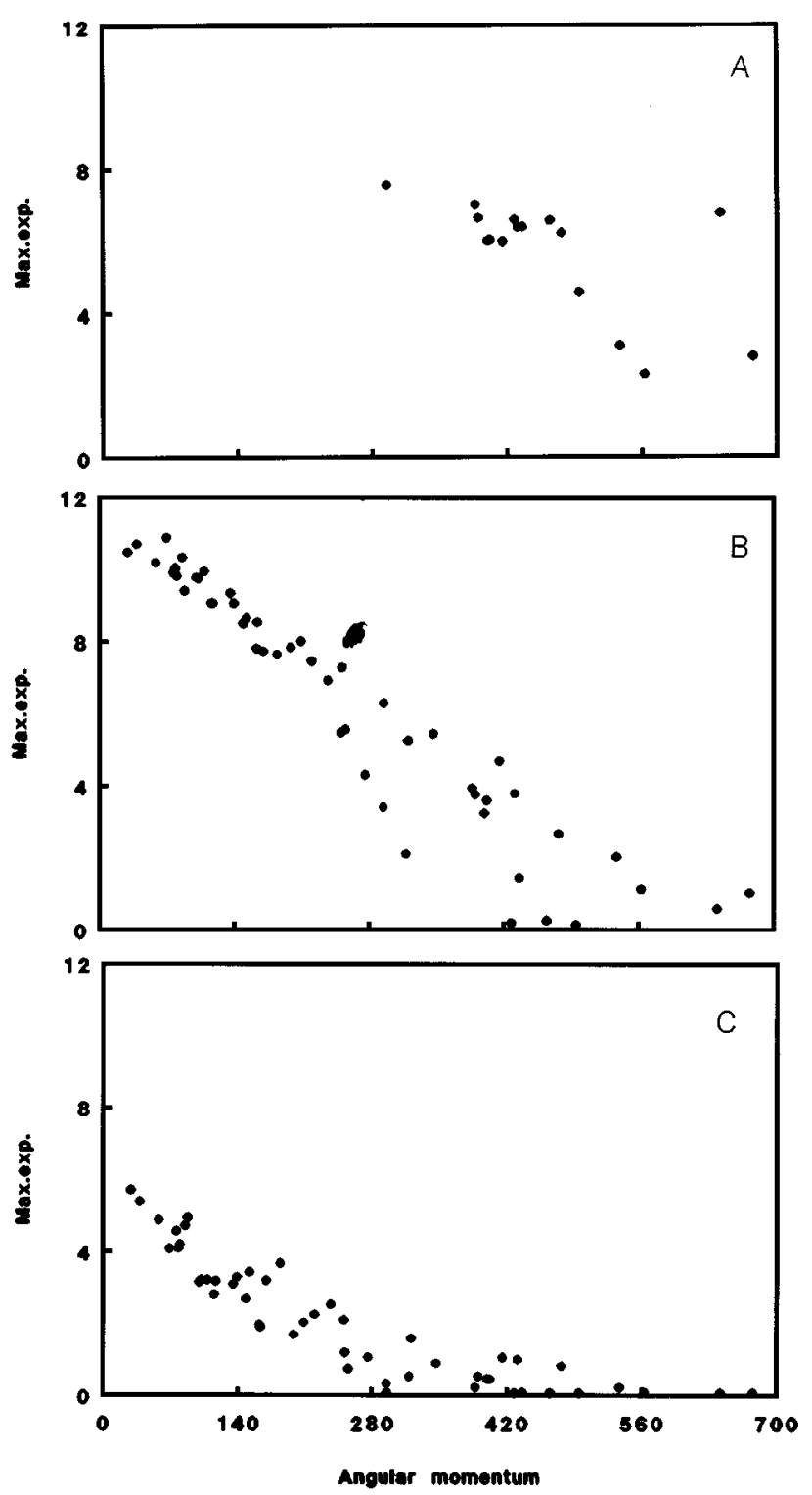

FIG. 5. Distribution of maximum Lyapunov exponents as a function of the total angular momentum. (a) $E=-0.26$, (b) $E=$ -0.65 , (c) $E=-0.80$.

equal vibrational kinetic energy values. As a result, the deviations of individual exponents are small. However, for rotating clusters the assigned vibrational kinetic energy within the ensemble varies drastically, and consequently the standard deviations are larger. The largest deviations are observed around the saddle-point range. As the energy gets either very low or very high, these deviations become relatively smaller. The linear clusters that are not depicted here display very similar behavior.

In order to carry out a further analysis of the effects of the angular momentum, we plot at three different energy values the maximum Lyapunov exponents as functions of the total angular momentum, which is a conserved quantity (Fig. 5). At $E=-0.26$ there are few nondissociating trajectories because low angular momenta trajectories tend to kick out a single atom before the Lyapunov exponent converges. Therefore, in order to reach a conclusion at low energies, a larger sample is required. On the other hand, the other two
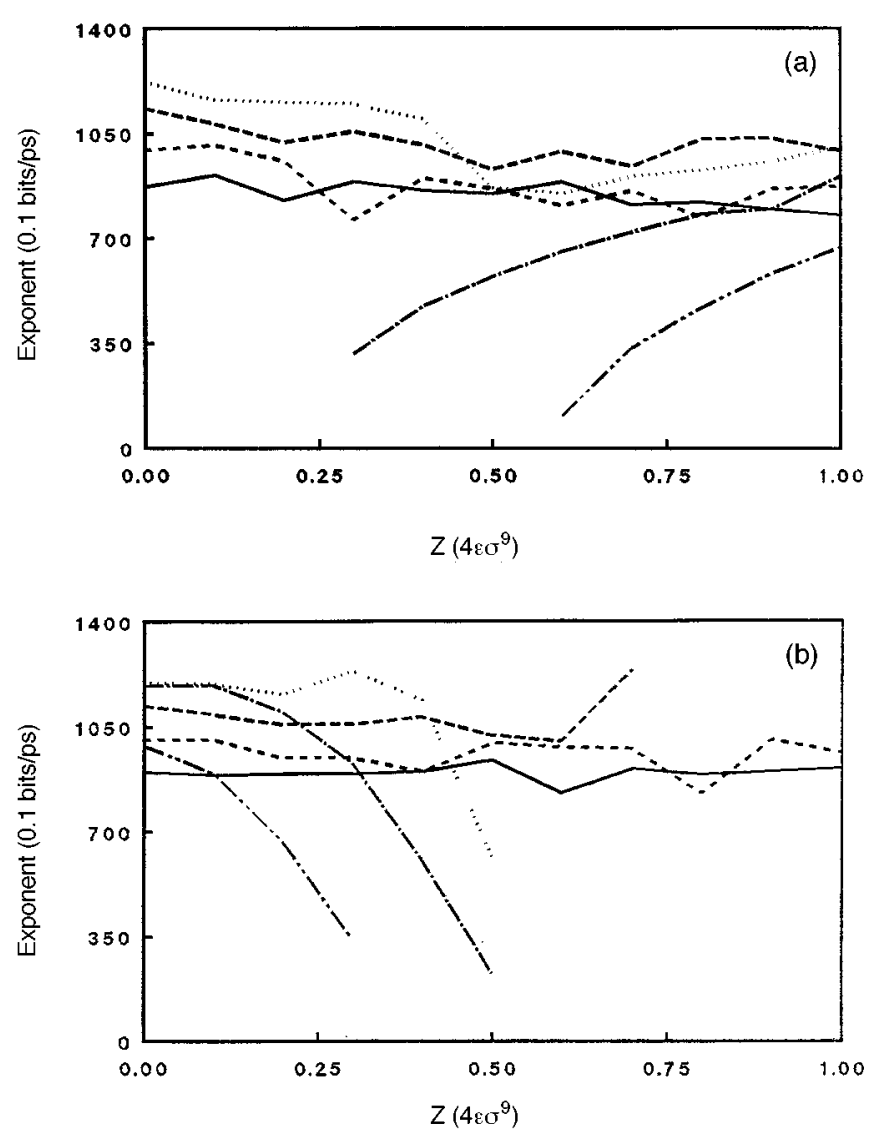

FIG. 6. Maximum Lyapunov exponent as a function of the Axilrod-Teller strength parameter $Z$. (a) Nonrotating linear cluster. (b) Nonrotating triangular cluster. - $-E=-0.40$; ----, $E=$ $-0.50 ;---, E=-0.60 ; \quad \ldots, E=-0.65 ;-.-\cdot, \quad E=-0.70$; $-\cdots-\cdot \cdot E=-0.75$.

figures at $E=-0.65$ and $E=-0.80$ display clearly the trend that high angular momentum generates lower Lyapunov exponents. Even though the coupling between rotational and vibrational motion should be larger for high angular momentum cases, again the magnitude of the initial vibrational kinetic energy seems to be the major factor for the determination of chaos. The work with employing larger ensembles is in progress.

\section{B. Three-body forces}

The introduction of three-body interactions into the Hamiltonian increases the nonlinearity of the system and one expects a higher degree of chaos. The strength of three-body interactions is controlled by the reduced parameter $Z$ whose value is assumed to change between 0 and 1 . Obviously the relative magnitudes of two- and three-body forces do not remain constant during the integration time and they change according to the instantaneous geometry of the cluster. The limiting values of the angular part of the Axilrod-Teller potential are -2 and $\frac{11}{8}$ for the linear and equilateral triangle, respectively (in reduced units). After $Z=0.5$ the linear cluster becomes the stable form. However, this initial partitioning of the potential energy into two- and three-particle interactions seems to play an important role in the measure of chaotic behavior. In Figs. 6(a) and 6(b), we present the variation of the maximum Lyapunov exponent as a function of the 


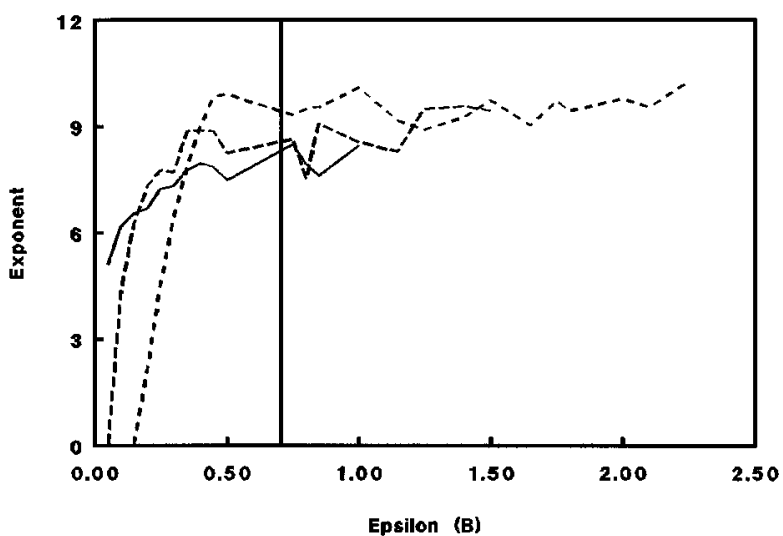

FIG. 7. Lyapunov exponent as a function of $\epsilon_{B} .-, E=$ $-0.40 ;----, E=-0.50 ; \ldots, E=-0.60$.

interaction strength $Z$ for nonrotating clusters. At highenergy values $E>-0.7$ the maximum Lyapunov exponent seems to be unaffected by the variation of $Z$. Once the energy is below the saddle point for the Lennard-Jones potential surface, then linear and triangular clusters show different behavior. Clusters start initially at linear geometry (stable form for $Z>0.5$ ), the chaotic behavior increases with increasing $Z$ whereas the clusters starting at triangular geometry show the opposite trend. Here it seems that the initial geometry of the cluster plays an important role in a somewhat unexpected way; that increasing nonlinearity does not necessarily increase the extent of the chaos. A larger set of calculations to study this problem in detail is under way.

\section{Heterogeneous clusters}

In this part of the work, parameters corresponding to one of the atoms are modified to generate heterogeneous clusters. Starting with a nonrotating cluster of triangular structure, the Lennard-Jones parameters $\epsilon$ and $\sigma$, and mass of one atom are changed and the effects on chaotic behavior are observed. The interaction parameters for unlike atoms are computed by standard Berhelot rules of combination. The starting geometry is the minimum-energy conformation of the equilateral triangle except for the variation of $\sigma$, where a simple optimization of the geometry is carried out for each case before

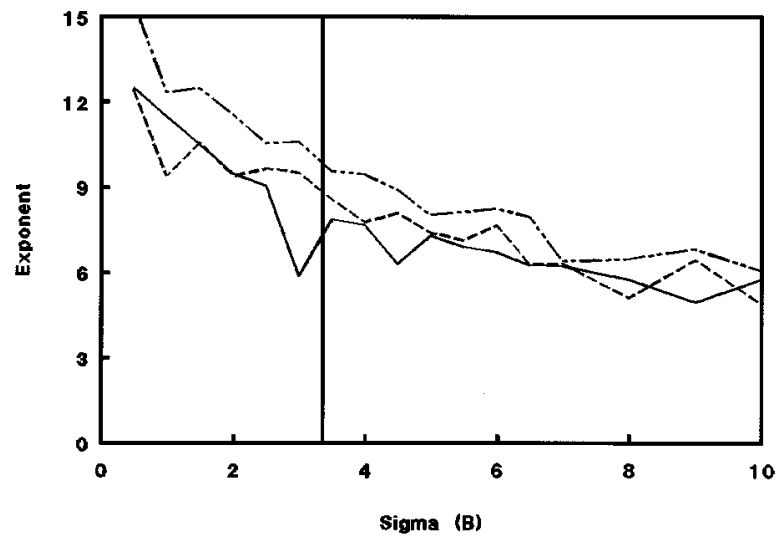

FIG. 8. Lyapunov exponent as a function of $\sigma_{B}$. $-E=$ $-0.40 ;----, E=-0.50 ; \ldots, E=-0.60$.

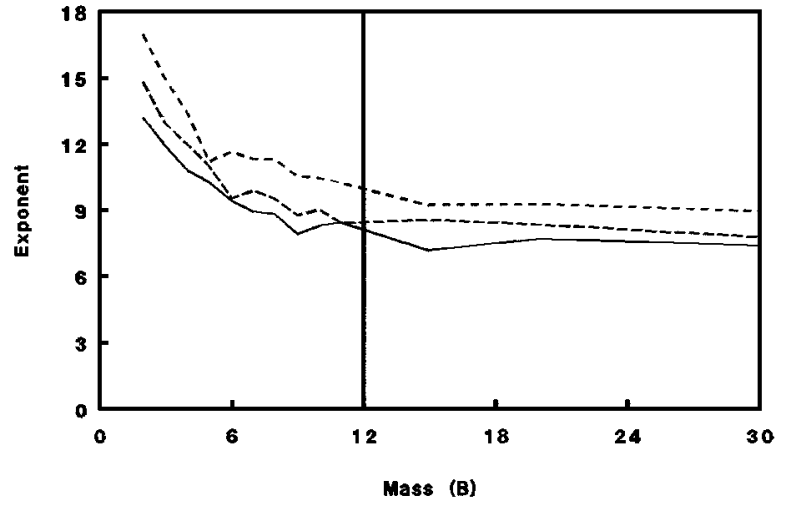

FIG. 9. Lyapunov exponent as a function of $m_{B}$.,$E=$ $-0.40 ;----, E=-0.50 ; \ldots, E=-0.60$.

the integration of equations of motion. In Figs. 7-9 these variations at different energy values are presented. The standard values of $\epsilon, \sigma$, and mass are noted by vertical lines in figures. When the interaction strength is varied, the results can be summarized as follows. Let us denote two equivalent atoms as $A$ and the third, different one, as $B$. If one of the atoms has a relatively smaller $\epsilon$, then the motion is quite regular. Such a system is equivalent to a diatomic cluster where the effect of the third particle is negligible and the regular dynamics prevails. This behavior is observed until the ratio of $\epsilon_{B} / \epsilon_{A}$ is around 0.7. Above this threshold, Lyapunov exponents remain reasonably constant even for ratios as large as 4, implying that there is no additional degree of chaos. According to Berthelot rules, the interaction strengths between unlike particles are calculated by geometric averages. Due to this slow change in off-diagonal interactions, one may expect, again, a relative decrease in chaotic behavior at very high ratios. On the other hand, upon increasing the $\sigma_{B} / \sigma_{A}$ ratio, we observe a gradual but continuous decrease. As one of the particles gets larger, the motion becomes relatively more regular but not to the extent that chaos disappears. The limiting case for such systems is the diatomic molecule on a surface, where if the surface is sufficiently large then the motions of the diatomic molecule and the surface are separable and again a regular dynamics will be observed. Finally, the effects of varying the mass of a particle are parallel to those of varying the size. Asymptotically an integrable system is going to be obtained since the motion of the heavy particle will almost stop and a practically one-dimensional motion should be observed.

In conclusion we would like to state that an increase in the nonlinearity of the interactions in a triatomic cluster does not necessarily increase the degree of the chaotic behavior. The major contributions to the chaotic motion come from the energy stored in vibrational modes. The extent of the energy in those modes classifies dynamics.

\section{ACKNOWLEDGMENTS}

We would like to acknowledge a critical reading of the manuscript by Gary Moore. This project was partially supported by the Volkswagen Stiftung Project No. I69/797. 
[1] On Clusters and Clustering, edited by P. J. Reynolds (NorthHolland, Amsterdam, 1993).

[2] Clusters and Colloids, edited by G. Schmid (VCH, Weinheim, 1994).

[3] S. Sugano, in Microcluster Physics (Springer-Verlag, Heidelberg, 1991).

[4] Clusters of Atoms and Molecules I, edited by H. Haberland (Springer-Verlag, Heidelberg, 1995).

[5] Clusters of Atoms and Molecules II, edited by H. Haberland (Springer-Verlag, Heidelberg, 1994).

[6] K. Raghavachari and J. S. Binkley, J. Chem. Phys. 87, 2191 (1987).

[7] D. J. Wales and R. S. Berry, J. Chem. Phys. 92, 4283 (1990).

[8] R. N. Barnett, U. Landman, A. Nitzan, and G. Rajagopal, J. Chem. Phys. 94, 608 (1991).

[9] D. G. Vlachos, L. D. Schmidt, and R. Aris, J. Chem. Phys. 96, 6880 (1992); 96, 6891 (1992).

[10] D. Zajfman, D. Kella, O. Heber, D. Majer, H. Feldman, Z. Vager, and R. Naaman, Z. Phys. D 26, 343 (1993).

[11] R. S. Dumont and S. Jain, J. Chem. Phys. 103, 6151 (1995).

[12] D. I. Zhukhovitskii, J. Chem. Phys. 103, 9401 (1995).

[13] C. Chakravarty, J. Chem. Phys. 103, 10663 (1995).
[14] T. Raz, U. Even, and R. D. Levine, J. Chem. Phys. 103, 5394 (1995).

[15] W. G. Hoover, H. A. Posch, and S. Bestiale, J. Chem. Phys. 87, 6665 (1987).

[16] H. A. Posch and W. G. Hoover, Phys. Rev. A 38, 473 (1988); H. A. Posch, W. G. Hoover, and B. L. Holian, Ber. Bunsenges. Phys. Chem. 94, 250 (1990).

[17] H. A. Posch and W. G. Hoover, Phys. Rev. A 39, 2175 (1989); T. L. Beck, D. M. Leitner, and R. S. Berry, J. Chem. Phys. 89, 1681 (1988).

[18] D. J. Wales and R. S. Berry, J. Phys. B 24, L351 (1991).

[19] R. J. Hinde, R. S. Berry, and D. J. Wales, J. Chem. Phys. 96, 1376 (1992).

[20] C. Amitrano and R. S. Berry, Phys. Rev. Lett. 68, 729 (1992).

[21] C. Amitrano and R. S. Berry, Phys. Rev. E 47, 3158 (1993).

[22] C. Amitrano and R. S. Berry, Z. Phys. D 26, 388 (1993).

[23] S. F. Chekmarev and F. S. Liu, Z. Phys. D 20, 231 (1991).

[24] E. Yurtsever and N. Elmac1, Ber. Bunsenges. Phys. Chem. 95, 467 (1991).

[25] D. J. Tildesley and P. A. Madden, Mol. Phys. 42, 1137 (1981).

[26] T. Halıcıoğlu, Chem. Phys. Lett. 179, 159 (1991). 